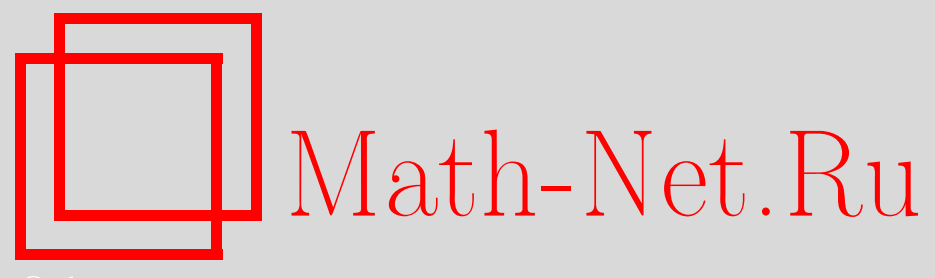

А. В. Нагаев, В. Р. Стебловская, О двумерной бинарной модели финансового рынка и ее обобщении, Дискрет. матем., 2006, том 18, выпуск 2, 3-28

DOI: https://doi.org/10.4213/dm43

Использование Общероссийского математического портала Math-Net.Ru подразумевает, что вы прочитали и согласны с пользовательским соглашением http://www . mathnet.ru/rus/agreement

Параметры загрузки:

IP : 35.173 .219 .149

26 апреля 2023 г., 15:02:05 


\title{
О двумерной бинарной модели финансового рынка и ее обобщении
}

\author{
() 2006 г. А. В. Нагаев, В. Р. Стебловская
}

\begin{abstract}
Рассматривается неполный рынок с европейским финансовым обязательством, построенным на двух акциях, эволюция цен которых носит бинарный характер. Получены явные формулы для нижней и верхней границ интервала справедливых цен, а также для верхних и нижних хеджирующих стратегий. Полученные стратегии являются полу-самофинансируемыми в том смысле, что на каждом шаге с неотрицательной вероятностью возможно извлечение капитала. Результат обобщается на случай, когда распределение скачков последовательных цен акций сосредоточено на замкнутом прямоугольнике, а платежная функция выпукла. Не делается никаких предположений о совместном распределении скачков цен.
\end{abstract}

\section{1. Введение}

Бинарная модель финансового рынка Кокса-Росса-Рубинштейна (КРР-модель, см. [1]) показала себя достаточно практичной при оценке стоимости финансовых опционов. Несмотря на ее простоту, эта модель достаточно реалистична, чтобы получать полезные оценки стоимости различных финансовых опционов. Эти факты стимулировали в последние десятилетия интенсивные исследования, цель которых состояла в таком обобщении КРР-модели, которое сохраняло бы простоту и достоинства этой модели, но устраняло ненужные ограничения.

Упомянем только некоторые работы наиболее тесно связанные с представленным в данной работе подходом. В [8] рассматривалась мультиномиальная модель. Эта модель обобщает КРР-модель. В ней скачки последовательных цен акции могут принимать несколько значений внутри некоторого интервала. Соответствующий рынок, в отличие от КРР-рынка, является неполным. Авторы определяют верхние справедливые цены европейского и американского опционов в терминах решений соответствующих задач управления.

Подход, разработанный в [8], был взят за основу в ряде работ А. В. Нагаева и др. $[4,5,6]$. В этих работах была предложена и исследована модель, в которой распределение скачков последовательных цен акции сосредоточено на замкнутом интервале. В $[5,6]$ изучались асимптотические свойства доходов или потерь инвестора, связанных с неполнотой рынка.

Другое направление обобщения КРР-модели представлено в [3]. Здесь рассматривались финансовые опционы, связанные с несколькими рисковыми активами. Процесс цен для каждого актива предполагался бинарным и независимым от процессов цен других 
активов. Авторы разработали два подхода для оценки опционов и хеджирования: геометрический и аналитический. Геометрический подход позволяет получить общие формулы для верхних справедливых цен опционов в терминах итераций некоторых преобразований в пространстве борелевских вешественных измеримых функций, заданных на $\mathbf{R}^{d}$. Важно, что авторы рассматривали только самофинансируемые хеджирующие стратегии. Аналитический подход использовался в случае, когда опцион построен на двух акщиях. Были получены явные формулы для верхней справедливой цены и верхнего хеджа на одношаговом бинарном рынке. Авторы показали также, что их результат можно обобщить на случай, когда скачки ограничены, а платежная функщия выпукла.

Целью предлагаемой статьи является дальнейшее обобщение модели КРР-рынка. Мы рассматриваем европейские платежные обязательства, построенные на двух акциях, эволюция цен которых носит бинарный характер. При таком подходе возникает неполнота рынка. Не делается никаких предположений о совместном распределении скачков цен, кроме предположения о том, что в каждом состоянии скачки с положительной вероятностью принимают одно из четырех значений в вершинах прямоугольника. Получены явные формулы для нижней и верхней границ интервала справедливых цен, а также для верхних и нижних хеджирующих стратегий. Поскольку интервал справедливых цен является открытым, то полученные верхние и нижние хеджирующие стратегии демонстрируют арбитражные возможности, возникающие на таком рынке для продавца и, соответственно, покупателя финансового обязательства. Мы не ограничиваемся самофинансируемыми хеджирующими стратегиями. На самом деле, полученные стратегии являются полу-самофинансируемыми в том смысле, что на каждом шаге с неотрицательной вероятностью возможно извлечение капитала. Результаты, полученные для бинарных процессов цен, обобщаются на случай, когда распределение скачков последовательных цен акций сосредоточено на замкнутом прямоугольнике, а платежная функщия является выпуклой. Это открывает возможности для дальнейшего обобщения модели, позволяющего учесть рисковые доходы или потери, связанные с неполнотой рынка, с использованием идей, развитых в $[5,6]$ для случая финансовых обязательств, построенных на одной акции.

Опишем структуру статьи. В разделе 2 приводится определение двумерной бинарной модели. В разделе 3 для начала исследуется двумерная бинарная одношаговая модель. Выводятся явные формулы как для верхней справедливой цены и отвечающего ей верхнего хеджирующего портфеля, так и соответствующие результаты для нижней справедливой цены. В разделе 4 эти результаты обобщаются естественным образом на двумерную бинарную динамическую (многошаговую) модель. Иллюстрируется существование арбитражных возможностей на границах интервала справедливых цен для одношаговой и многошаговой моделей. В разделе 5 эти результаты обобщаются на случай, когда распределение вектора скачков цен акций сосредоточено не на конечном множестве точек, а на замкнутом прямоугольнике. Полученные результаты справедливы для любого европейского платежного обязательства с выпуклой платежной функцией. В разделе 6 приводятся заключительные замечания.

\section{2. Модель}

Рассматривается финансовый рынок, на котором имеются два типа ценных бумаг. Для ценной бумаги первого типа эволюция цен задается соотношением

$$
b_{k}=b_{0} \rho^{k}, \quad k=0,1,2, \ldots,
$$


где $b_{0}>0, \rho \geqslant 1$. Значение $(\rho-1) \cdot 100 \%$ можно рассматривать как безрисковую процентную ставку, так что ценные бумаги этого типа являются безрисковыми. Условно мы будем называть их облигациями. Цены заданы в дискретные моменты времени $k=0,1,2, \ldots$ Без потери общности предполагается, что $b_{0}=1$.

Мы будем рассматривать две ценные бумаги второго типа. Для $i$-й ценной бумаги цена $s_{k, i}$ в момент $k$ задается соотношением

$$
s_{k, i}=s_{0, i} \prod_{l=1}^{k} \xi_{l, i}, \quad k=0,1,2, \ldots, \quad i=1,2,
$$

где скачки цен $\xi_{j, i}=s_{j, i} / s_{j-1, i}, i=1,2$, являются случайными величинами.

Очевидно, что при проведении операций с ценными бумагами второго типа нужно учитывать неопределенность их будущих цен. Такие рискованные ценные бумаги мы будем условно называть акциями.

Заданные количества $\beta, \gamma_{1}$ и $\gamma_{2}$ соответствующих ценных бумаг составляют портфель инвестора (или просто портфель), цена которого в момент $k$ равна $\beta b_{k}+\gamma_{1} s_{k, 1}+\gamma_{2} s_{k, 2}$. Игра на рассматриваемом финансовом рынке состоит в последовательном изменении содержания портфеля в моменты $k=1,2, \ldots, n-1$, где $n$ - момент исполнения платежного обязательства. Последовательные триплеты $\sigma(0)=\left(\beta_{0}, \gamma_{0,1}, \gamma_{0,2}\right), \sigma(1)=\left(\beta_{1}, \gamma_{1,1}, \gamma_{1,2}\right)$, $\ldots, \sigma(n-1)=\left(\beta_{n-1}, \gamma_{n-1,1}, \gamma_{n-1,2}\right)$ определяют стратегию торговли (или хеджирующую стратегию). Очевидно, что выбор триплета $\sigma(k)$ в момент времени $k$ зависит от эволюции цен акций до этого момента включительно, то есть $\beta_{k}=\beta_{k}\left(s^{(0)}, s^{(1)}, \ldots, s^{(k)}\right)$, $\gamma_{k, i}=\gamma_{k, i}\left(s^{(0)}, s^{(1)}, \ldots, s^{(k)}\right), i=1,2$, где $s^{(k)}=\left(s_{k, 1}, s_{k, 2}\right), k=0,1, \ldots, n-1$.

Под платежным обязательством $X$ мы будем в основном понимать опцион европейского типа, задающийся платежной функцией, которая зависит только от цен соответствующих акций в момент исполнения обязательства, то есть $X=f\left(s^{(n)}\right)$. Мы будем рассуждать с позиций продавца опциона (инвестора). Цель инвестора состоит в построении хеджирующей стратегии, удовлетворяющей условию

$$
x(n)=\beta_{n-1} b_{n}+\gamma_{n-1,1} s_{n, 1}+\gamma_{n-1,2} s_{n, 2} \geqslant f\left(s^{(n)}\right),
$$

где $f(s), s \in \mathbf{R}^{2}$ - платежная функция европейского опциона с датой исполнения $n$.

\section{3. Одношаговый двумерный бинарный рынок}

\section{1. Предварительные сведения, мартингальные меры}

Рассмотрим сначала одношаговый рынок, когда имеется только один период между началом торгов $k=0$ и датой исполнения $k=1$. Такой рынок определяется известными значениями $b_{0}, \rho$ и $s^{(0)}=\left(s_{0,1}, s_{0,2}\right)$ в начальный момент $k=0$ и неопределенными значениями цен $s^{(1)}=\left(s_{1,1}, s_{1,2}\right)$ в момент исполнения $k=1$. Предполагается, что рынок бинарен, то есть $\xi_{1}=s_{1,1} / s_{0,1} \in\left\{d_{1}, u_{1}\right\}, \xi_{2}=s_{1,2} / s_{0,2} \in\left\{d_{2}, u_{2}\right\}$, где $d_{i}<\rho<u_{i}$, $i=1,2$. Вектор $\xi=\left(\xi_{1}, \xi_{2}\right)$ - это случайный вектор на некотором выборочном пространстве, которое мы сейчас опишем.

Рассмотрим конечное выборочное пространство $\Omega^{(1)}=\left\{\omega_{1}^{(1)}, \omega_{2}^{(1)}, \omega_{3}^{(1)}, \omega_{4}^{(1)}\right\}$, где $\omega_{j}^{(1)}, j=1,2,3,4$, представляет собой одно из возможных состояний природы, которое неизвестно и момент $k=0$ и становится известным в момент $k=1$. В нашем случае 
$\omega_{j}^{(1)}$ - одно из возможных значений вектора скачков $\xi=\left(\xi_{1}, \xi_{2}\right)$, то есть

$$
\omega_{1}^{(1)}=\left(u_{1}, u_{2}\right), \quad \omega_{2}^{(1)}=\left(u_{1}, d_{2}\right), \quad \omega_{3}^{(1)}=\left(d_{1}, u_{2}\right), \quad \omega_{4}^{(1)}=\left(d_{1}, d_{2}\right) .
$$

Рассмотрим стратегию торговли $\sigma=\left(\beta, \gamma_{1}, \gamma_{2}\right)$. Она определяет начальный портфель стоимостью

$$
x(0)=\beta b_{0}+\gamma_{1} s_{0,1}+\gamma_{2} s_{0,2}
$$

В момент исполнения $k=1$ стоимость портфеля равна

$$
x(1)=\beta b_{1}+\gamma_{1} s_{1,1}+\gamma_{2} s_{1,2}=\beta b_{0} \rho+\gamma_{1} s_{0,1} \xi_{1}+\gamma_{2} s_{0,2} \xi_{2}
$$

Инвестор (продавец опциона) выполнит свои обязательства с вероятностью 1 если

$$
x(1) \geqslant f\left(s^{(1)}\right),
$$

что соответствует соотношению (2.1) для одношагового случая.

Рынок является полным, если для любого платежного обязательства $X$ существует реплицирующая стратегия торговли, то есть стратегия, для которой стоимость портфеля в момент исполнения платежного обязательства совпадает с платежной функцией этого обязательства. В частности, на полном рынке для любого платежного обязательства вида $X=f\left(s^{(1)}\right)$ существует стратегия торговли, ведущая к равенству

$$
x(1)=f\left(s^{(1)}\right) .
$$

Мы не накладываем никаких условий на совместное распределение величин $\xi=\left(\xi_{1}, \xi_{2}\right)$. Единственное ограничение состоит в том, что каждое из значений $\omega_{j}^{(1)}, j=1,2,3,4$, принимается величиной $\xi$ с положительной вероятностью. Из этого ограничения вытекает, что рассматриваемый рынок является неполным: в этом случае условие (3.2) эквивалентно системе четырех линейных уравнений с тремя неизвестными. А значит,

(a) существует платежное обязательство $X=f\left(s^{(1)}\right)$, для которого не существует такой стратегии торговли, что выполняется (3.2), и, как мы показываем ниже,

(б) множество нейтральных к риску мер не является одноточечным.

Определение 1. Вероятностная мера $Q$ называется нейтральной к риску, если:

(а) ее значение положительно на каждом из состояний $\omega_{j}^{(1)}, j=1,2,3,4$, и

(б) справедливы равенства

$$
\mathbf{E}_{Q} \xi_{i}=\rho, \quad i=1,2,
$$

(см., например, раздел 1.3 в [7]).

Здесь $\mathbf{E}_{Q}$ обозначает математическое ожидание относительно меры $Q$.

Условие (а) приведенного выше определения означает, что мера $Q$ эквивалентна любой строго положительной вероятностной мере на $\Omega^{(1)}$. Условие (3.3) означает, что мера 
$Q$ является мартингальной мерой, то есть такой мерой, относительно которой дисконтированные цены акций $s_{k, i}^{*}=s_{k, i} / b_{k}, i=1,2, k=0,1$, образуют мартингал. В самом деле, условие (3.3) эквивалентно тому, что $\mathbf{E}_{Q}\left(s_{1, i}^{*}\right)=s_{0, i}^{*}, i=1,2$.

Мы хотим найти нейтральную к риску меру $Q$, которая соответствует случаю одношагового двумерного бинарного рынка. Начнем с поиска вероятностной меры, удовлетворяющей соотношению (3.3). Введем обозначения

$$
Q\left(u_{1}, u_{2}\right)=q_{1}, \quad Q\left(u_{1}, d_{2}\right)=q_{2}, \quad Q\left(d_{1}, u_{2}\right)=q_{3}, \quad Q\left(d_{1}, d_{2}\right)=q_{4} .
$$

Из (3.3) получаем, что

$$
\begin{aligned}
u_{1}\left(q_{1}+q_{2}\right)+d_{1}\left(q_{3}+q_{4}\right) & =\rho, \\
u_{2}\left(q_{1}+q_{3}\right)+d_{2}\left(q_{2}+q_{4}\right) & =\rho, \\
q_{1}+q_{2}+q_{3}+q_{4} & =1, \\
q_{1}, q_{2}, q_{3}, q_{4} & \geqslant 0 .
\end{aligned}
$$

Решением системы уравнений (3.4) является однопараметрическое семейство 2 вероятностных мер $Q_{\lambda}$ вида

$$
\begin{aligned}
& Q_{\lambda}\left(u_{1}, u_{2}\right)=\lambda, \quad Q_{\lambda}\left(u_{1}, d_{2}\right)=p_{1}-\lambda, \\
& Q_{\lambda}\left(d_{1}, u_{2}\right)=p_{2}-\lambda, \quad Q_{\lambda}\left(d_{1}, d_{2}\right)=1-p_{1}-p_{2}+\lambda \text {. }
\end{aligned}
$$

Здесь

$$
p_{i}=\frac{\rho-d_{i}}{u_{i}-d_{i}}, \quad i=1,2,
$$

и параметр $\lambda$ удовлетворяет неравенствам

$$
\lambda_{-}=\max \left(0, p_{1}+p_{2}-1\right) \leqslant \lambda \leqslant \min \left(p_{1}, p_{2}\right)=\lambda_{+} .
$$

Всюду далее мы предполагаем, что $0<p_{i}<1, i=1,2$, а поэтому выполняется неравенство

$$
\max \left(0, p_{1}+p_{2}-1\right)<\min \left(p_{1}, p_{2}\right)
$$

Замечание 1. Заметим, что все меры из множества 2, за исключением двух специальных мер $Q_{\lambda_{+}}$и $Q_{\lambda_{-}}$, являются эквивалентными мартингальными мерами, а значит, они нейтральны к риску. Меры $Q_{\lambda_{+}}$и $Q_{\lambda_{-}}$сосредоточены на трех или двух, а не на четырех точках выборочного пространства (см. приводимые ниже таблицы).

\begin{tabular}{|c|c|c|c|}
\hline & $p_{1}<p_{2}$ & $p_{1}>p_{2}$ & $p_{1}=p_{2}=p$ \\
\hline$Q_{\lambda_{+}}$ & $\left(p_{1}, 0, p_{2}-p_{1}, 1-p_{2}\right)$ & $\left(p_{2}, p_{1}-p_{2}, 0,1-p_{1}\right)$ & $(p, 0,0,1-p)$ \\
\hline
\end{tabular}

\begin{tabular}{|c|c|c|c|}
\hline & $p_{1}+p_{2}<1$ & $p_{1}+p_{2}>1$ & $p_{1}+p_{2}=1$ \\
\hline$Q_{\lambda_{-}}$ & $\left(0, p_{1}, p_{2}, 1-p_{1}-p_{2}\right)$ & $\left(p_{1}+p_{2}-1,1-p_{2}, 1-p_{1}, 0\right)$ & $\left(0, p_{1}, p_{2}, 0\right)$ \\
\hline
\end{tabular}

Они не удовлетворяют условию (а) определения 1, а значит, не являются нейтральными к риску. Будем называть эти неэквивалентные мартингальные меры верхней (соответственно нижней) мартингальной мерой. 


\section{2. Верхняя справедливая цена}

Введем сначала следующие обозначения: для любой пары $s=\left(s_{1}, s_{2}\right) \in \mathbf{R}_{+}^{2}$, положим

$$
\begin{array}{ll}
f_{++}(s)=f\left(s_{1} u_{1}, s_{2} u_{2}\right), & f_{+-}(s)=f\left(s_{1} u_{1}, s_{2} d_{2}\right), \\
f_{-+}(s)=f\left(s_{1} d_{1}, s_{2} u_{2}\right), & f_{--}(s)=f\left(s_{1} d_{1}, s_{2} d_{2}\right) .
\end{array}
$$

Определим далее

$$
d_{f}(s)=f_{++}(s)+f_{--}(s)-f_{+-}(s)-f_{-+}(s)
$$

Положим для краткости

$$
\begin{aligned}
f_{++} & =f_{++}\left(s^{(0)}\right), \quad f_{+-}=f_{+-}\left(s^{(0)}\right), \quad f_{-+}=f_{-+}\left(s^{(0)}\right), \\
f_{--} & =f_{--}\left(s^{(0)}\right),
\end{aligned}
$$

где $s^{(0)}$ - вектор начальных цен акций. Далее, для любой функции $f$, принимающей действительные значения и определенной на $\mathbf{R}_{+}^{2}$, определим множества

$$
\begin{aligned}
& S_{+}=\left\{s: s \in \mathbf{R}_{+}^{2}, d_{f}(s)>0\right\} \\
& S_{-}=\left\{s: s \in \mathbf{R}_{+}^{2}, d_{f}(s)<0\right\} \\
& S_{0}=\left\{s: s \in \mathbf{R}_{+}^{2}, d_{f}(s)=0\right\}
\end{aligned}
$$

В качестве примера рассмотрим платежную функцию европейского пакетного опциона покупки с ценой исполнения $K$

$$
f(s)=\left(a_{1} s_{1}+a_{2} s_{2}-K\right)_{+},
$$

где $a_{1}>0, a_{2}>0, a_{1}+a_{2}=1$ и $K>0$. Нетрудно проверить, что для этой функции

$$
S_{+}=\left\{s: s \in \mathbf{R}_{+}^{2}, a_{1} d_{1} s_{1}+a_{2} d_{2} s_{2}<K, a_{1} u_{1} s_{1}+a_{2} u_{2} s_{2}>K\right\},
$$

$S_{0}=\mathbf{R}_{+}^{2} \backslash S_{+}$и $S_{-}=\varnothing$.

Верхняя справедливая цена платежного обязательства - это минимальный капитал в момент $k=0$, который позволяет инвестору выполнить обязательства по контракту с вероятностью 1 . Обозначим через $\bar{x}(0)$ верхнюю справедливую цену платежного обязательства $X=f\left(s^{(1)}\right)$. Известно, что

$$
\bar{x}(0)=\rho^{-1} \sup _{Q \in \mathcal{Q}} \mathbf{E}_{Q} f\left(s^{(1)}\right)
$$

(см, например, утверждение 1.28 в [7]). Заметим, что величина $\bar{x}(0)$ является функщией от начальных цен $s^{(0)}$, поскольку $s^{(1)}=\left(s_{0,1} \xi_{1}, s_{0,2} \xi_{2}\right)$.

Для вычисления верхней справедливой цены $\bar{x}(0)$ подсчитаем сначала математическое ожидание в (3.11). Для любой меры $Q \in 2$ получаем равенство

$$
\begin{aligned}
\mathbf{E}_{Q f\left(s^{(1)}\right)} & =\mathbf{E}_{Q} f\left(s_{0,1} \xi_{1}, s_{0,2} \xi_{2}\right) \\
& =f_{++} \lambda+f_{+-}\left(p_{1}-\lambda\right)+f_{-+}\left(p_{2}-\lambda\right)+f_{--}\left(1-p_{1}-p_{2}+\lambda\right) \\
& =d_{f} \lambda+f_{+-} p_{1}+f_{-+} p_{2}+f_{--}\left(1-p_{1}-p_{2}\right) .
\end{aligned}
$$


Теперь надо найти супремум правой части соотношения (3.12) по всем допустимым значениям $\lambda$ и подставить его в соотношение (3.11). Очевидно, что значение упомянутого супремума зависит от знака величины $d_{f}$. А именно, если $d_{f}>0$ (или, что эквивалентно, если $s^{(0)} \in S_{+}$), то (3.12) достигает своего максимального значения, когда $\lambda$ максимально, именно, $\lambda=\lambda_{+}$(см. (3.7)); если $d_{f}<0$ (или, что эквивалентно, если $s^{(0)} \in S_{-}$), то (3.12) достигает своего максимального значения, когда $\lambda$ минимально, именно, $\lambda=\lambda_{-}$; наконец, если $d_{f}=0$ (или, что эквивалентно, если $s^{(0)} \in S_{0}$ ), то математическое ожидание (3.12) не зависит от $\lambda$. Из приведенных наблюдений вытекает, что в случае $s^{(0)} \in S_{+}$верхняя справедливая цена $\bar{x}(0)$ задается соотношением

$$
\bar{x}(0)=\rho^{-1} \mathbf{E}_{Q_{\lambda_{+}}} f\left(s^{(1)}\right),
$$

в то время как в случае $s^{(0)} \in S_{-}$она задается соотношением

$$
\bar{x}(0)=\rho^{-1} \mathbf{E}_{Q_{\lambda-}} f\left(s^{(1)}\right) .
$$

Мы знаем (см. замечание 1), что верхняя (соответственно нижняя) мартингальная мера $Q_{\lambda_{+}}$(соответственно $Q_{\lambda_{-}}$) не является нейтральной к риску. Этот факт будет рассмотрен позже в разделе 3.5. А пока мы просто используем приведенные соотношения (3.13) и (3.14) для подсчета верхней справедливой цены.

Удобно ввести следующие обозначения:

$$
\begin{aligned}
& x_{++}=\frac{1}{\rho}\left(p_{1} f_{+-}+p_{2} f_{-+}+\left(1-p_{1}-p_{2}\right) f_{--}\right), \\
& x_{+-}=\frac{1}{\rho}\left(p_{1} f_{++}+\left(p_{2}-p_{1}\right) f_{-+}+\left(1-p_{2}\right) f_{--}\right), \\
& x_{-+}=\frac{1}{\rho}\left(p_{2} f_{++}+\left(p_{1}-p_{2}\right) f_{+-}+\left(1-p_{1}\right) f_{--}\right), \\
& x_{--}=\frac{1}{\rho}\left(\left(p_{1}+p_{2}-1\right) f_{++}+\left(1-p_{2}\right) f_{+-}+\left(1-p_{1}\right) f_{-+}\right) .
\end{aligned}
$$

Выбирая соответствуюшие значения параметра $\lambda$ из (3.7) и вычисляя выражения (3.13) и

\begin{tabular}{|c|c|c|c|c|}
\hline \multirow{2}{*}{$s^{(0)} \in S_{-}$} & $p_{1}+p_{2}<1$ & \multicolumn{2}{|c|}{$p_{1}+p_{2}>1$} & $p_{1}+p_{2}=1$ \\
\hline & $x++$ & \multicolumn{2}{|c|}{$x$} & $x_{++}=x_{--}$ \\
\hline & $p_{1}<p_{2}$ & $p_{1}>p_{2}$ & & $=p_{2}=p$ \\
\hline$s^{(0)} \in S$ & $x_{+-}$ & $x_{-+}$ & & $-=x_{-+}$ \\
\hline
\end{tabular}
(3.14) для каждого из этих значений, мы приходим к следуюшему предложению.

Предложение 1. Пусть $X=f\left(s^{(1)}\right)$ - платежное обязательство, и пусть множества $S_{+}, S_{-}$u $S_{0}$ определены в (3.9) для платежной функции $f$.

(а) Если $s^{(0)} \in S_{-}$или $s^{(0)} \in S_{+}$, то верхняя справедливая чена $\bar{x}(0)$ платежного обязательства $X$ в момент $k=0$ задается следующими таблицами:

(б) Если $s^{(0)} \in S_{0}$, то существует единственная верхняя справедливая цена платежного обязательства $Х$ в момент $k=0$, равная $x_{++}=x_{+-}=x_{-+}=x_{--}$. 
Доказательство. Остается рассмотреть случай $s^{(0)} \in S_{0}$ (или $\left.d_{f}=0\right)$. Из (3.11)-(3.12) следует, что в этом случае

$$
\bar{x}(0)=\rho^{-1} \mathbf{E}_{Q_{\lambda}} f\left(s^{(1)}\right)=\rho^{-1}\left(f_{+-} p_{1}+f_{-+} p_{2}+f_{--}\left(1-p_{1}-p_{2}\right)\right) .
$$

Здесь $Q=Q_{\lambda}-$ любая мера из множества 2, и результат не зависит от значения $\lambda$. Легко проверить, что в этом случае каждое из выражений (3.15)-(3.18) сводится к (3.19).

Замечание 2. В разделе 3.1 мы нашли множество мартингальных мер на выборочном пространстве $\Omega^{(1)}$, а в разделе 3.2 - верхнюю границу для интервала справедливых цен. Эти две задачи можно объединить в одну задачу линейного программирования (ЛП) следующим образом:

$$
\text { максимизировать } Y_{1} f_{++}+Y_{2} f_{+-}+Y_{3} f_{-+}+Y_{4} f_{--}
$$

при условиях

$$
\begin{aligned}
u_{1}\left(Y_{1}+Y_{2}\right)+d_{1}\left(Y_{3}+Y_{4}\right) & =1 \\
u_{2}\left(Y_{1}+Y_{3}\right)+d_{2}\left(Y_{2}+Y_{4}\right) & =1 \\
Y_{1}+Y_{2}+Y_{3}+Y_{4} & =1 / \rho \\
Y_{1}, Y_{2}, Y_{3}, Y_{4} & \geqslant 0 .
\end{aligned}
$$

При $Y_{j}=q_{j} / \rho$ максимизация целевой функции (3.20) эквивалентна поиску величины $\bar{x}(0)$ из соотношения (3.11), а ограничения, определяющие допустимую область (3.21), превращаются в (3.4).

\section{3. Верхние хеджирующие стратегии}

Займемся теперь стратегиями торговли, которые отвечают соответствующим верхним справедливым ценам (3.15)-(3.18). Такие стратегии называются также верхними хеджирующими стратегиями или просто верхними хеджами. Напомним, что стратегия торговли - это триплет $\sigma=\left(\beta, \gamma_{1}, \gamma_{2}\right)$, где $\beta$ - число облигаций в портфеле инвестора, в то время как $\gamma_{i}, i=1,2$, - это число $i$-х акций в этом портфеле. Задача верхнего хеджирования может быть сформулирована в виде следующей задачи ЛП:

$$
\text { минимизировать } \beta b_{0}+\gamma_{1} s_{0,1}+\gamma_{2} s_{0,2}
$$

при условии

$$
\begin{aligned}
& \beta b_{0} \rho+\gamma_{1} s_{0,1} u_{1}+\gamma_{2} s_{0,2} u_{2} \geqslant f_{++}, \\
& \beta b_{0} \rho+\gamma_{1} s_{0,1} u_{1}+\gamma_{2} s_{0,2} d_{2} \geqslant f_{+-}, \\
& \beta b_{0} \rho+\gamma_{1} s_{0,1} d_{1}+\gamma_{2} s_{0,2} u_{2} \geqslant f_{-+}, \\
& \beta b_{0} \rho+\gamma_{1} s_{0,1} d_{1}+\gamma_{2} s_{0,2} d_{2} \geqslant f_{--} .
\end{aligned}
$$

Целевая функция (3.22) соответствует стоимости портфеля в момент времени $k=0$, a ограничения для допустимой области (3.23) соответствуют ограничениям (3.1), которые гарантируют, что инвестор может выполнить все обязательства по контракту с вероятностью 1. Легко проверить, что задача ЛП (3.20)-(3.21) (см. замечание 2) и задача ЛП (3.22)-(3.23) являются дуальными друг к другу. Мы нашли множество 2 мартингальных 
мер, которые дают оптимальное решение задачи (3.20)-(3.21). Поэтому, в силу теоремы дуальности линейного программирования (см., например, [7], с. 253) задача ЛП (3.22)(3.23) также имеет множество оптимальных решений, а соответствующие оптимальные значения целевых функционалов в сформулированных выше задачах ЛП совпадают.

Будем искать оптимальную стратегию $\sigma=\left(\beta, \gamma_{1}, \gamma_{2}\right)$ среди множества экстремальных стратегий, соответствующих вершинам области, отвечающей соотношению (3.23). Поступим следующим образом. Выберем три линейных неравенства в системе (3.23) и заменим их на соответствующие линейные равенства. Решим полученную систему линейных уравнений. После того, как решение $\sigma=\left(\beta, \gamma_{1}, \gamma_{2}\right)$ найдено, убедимся в его допустимости, проверяя выполнение оставшегося четвертого неравенства.

Начнем с решения системы

$$
\begin{aligned}
& \beta b \rho+\gamma_{1} s_{1} u_{1}+\gamma_{2} s_{2} u_{2}=f_{++}, \\
& \beta b \rho+\gamma_{1} s_{1} u_{1}+\gamma_{2} s_{2} d_{2}=f_{+-}, \\
& \beta b \rho+\gamma_{1} s_{1} d_{1}+\gamma_{2} s_{2} u_{2}=f_{-+} .
\end{aligned}
$$

Здесь $b, s_{1}$ и $s_{2}$ отвечают соответственно $b_{0}, s_{0,1}$ и $s_{0,2}$. Решая систему (3.24), получаем

$$
\gamma_{1}^{--}=\frac{f_{++}-f_{-+}}{s_{1}\left(u_{1}-d_{1}\right)}, \quad \gamma_{2}^{--}=\frac{f_{++}-f_{+-}}{s_{2}\left(u_{2}-d_{2}\right)}
$$

и

$$
\beta^{--}=\frac{1}{b \rho}\left(\left(1-\frac{u_{1}}{u_{1}-d_{1}}-\frac{u_{2}}{u_{2}-d_{2}}\right) f_{++}+\frac{u_{2}}{u_{2}-d_{2}} f_{+-}+\frac{u_{1}}{u_{1}-d_{1}} f_{-+}\right) .
$$

Замечание 3. Мы выбрали обозначение $\sigma^{--}=\left(\beta^{--}, \gamma_{1}^{--}, \gamma_{2}^{--}\right)$для полученной выше экстремальной стратегии, поскольку правая часть оставшегося четвертого неравенства равна $f_{-\ldots}$. Следуя этому принципу легко ввести обозначения для других экстремальных стратегий.

Вопрос о допустимости экстремальной стратегии $\sigma^{--}$решается проверкой выполнения ограничения, задаваемого четвертым неравенством в (3.23):

$$
b \rho \beta^{--}+s_{1} d_{1} \gamma_{1}^{--}+s_{2} d_{2} \gamma_{2}^{--} \geqslant f_{--} .
$$

Подстановка соответствующих выражений из (3.25) и (3.26) в последнее неравенство приводит к неравенству

$$
-f_{++}+f_{+-}+f_{-+} \geqslant f_{--}
$$

или, что эквивалентно, к неравенству $d_{f} \leqslant 0$. Отсюда следует, что стратегия $\sigma^{--}$допустима тогда и только тогда, когда $d_{f} \leqslant 0$.

Используя описанный подход, мы получим оставшиеся три оптимальные стратегии торговли $\sigma^{++}, \sigma^{+-}$и $\sigma^{-+}$. Их компоненты имеют вид

$$
\gamma_{1}^{++}=\frac{f_{+-}-f_{--}}{s_{1}\left(u_{1}-d_{1}\right)}, \quad \gamma_{2}^{++}=\frac{f_{-+}-f_{--}}{s_{2}\left(u_{2}-d_{2}\right)}
$$

и

$$
\beta^{++}=\frac{1}{b \rho}\left(-\frac{d_{1}}{u_{1}-d_{1}} f_{+-}-\frac{d_{2}}{u_{2}-d_{2}} f_{-+}+\left(1+\frac{d_{1}}{u_{1}-d_{1}}+\frac{d_{2}}{u_{2}-d_{2}}\right) f_{--}\right) ;
$$


Таблица 1.

\begin{tabular}{|c|c|c|c|}
\hline & $p_{1}+p_{2}<1$ & $p_{1}+p_{2}>1$ & $p_{1}+p_{2}=1$ \\
\hline$s^{(0)} \in S_{-}$ & $\left(x_{++} ; \sigma^{++}\right)$ & $\left(x_{--} ; \sigma^{--}\right)$ & $\left(x_{++}=x_{--} \sigma=\alpha \sigma^{++}+(1-\alpha) \sigma_{--}\right)$ \\
& & & $0 \leqslant \alpha \leqslant 1$ \\
\hline
\end{tabular}

$$
\gamma_{1}^{+-}=\frac{f_{++}-f_{-+}}{s_{1}\left(u_{1}-d_{1}\right)} . \quad \gamma_{2}^{+-}=\frac{f_{-+}-f_{--}}{s_{2}\left(u_{2}-d_{2}\right)}
$$

и

$$
\begin{gathered}
\beta^{+-}=\frac{1}{b \rho}\left(-\frac{d_{1}}{u_{1}-d_{1}} f_{++}+\left(\frac{d_{1}}{u_{1}-d_{1}}-\frac{d_{2}}{u_{2}-d_{2}}\right) f_{-+}+\frac{u_{2}}{u_{2}-d_{2}} f_{--}\right) ; \\
\gamma_{1}^{-+}=\frac{f_{+-}-f_{--}}{s_{1}\left(u_{1}-d_{1}\right)}, \quad \gamma_{2}^{-+}=\frac{f_{++}-f_{+-}}{s_{2}\left(u_{2}-d_{2}\right)}
\end{gathered}
$$

и

$$
\beta^{-+}=\frac{1}{b \rho}\left(-\frac{d_{2}}{u_{2}-d_{2}} \hat{i}+++\left(\frac{d_{2}}{u_{2}-d_{2}}-\frac{d_{1}}{u_{1}-d_{1}}\right) f_{+-}+\frac{u_{1}}{u_{1}-d_{1}} f_{--}\right) .
$$

Более того, оказывается, что стратегии $\sigma^{--}$и $\sigma^{++}$допустимы тогда и только тогда, когда $d_{f} \leqslant 0$, в то время как стратегии $\sigma^{+-}$и $\sigma^{-+}$допустимы тогда и только тогда, когда $d_{f} \geqslant 0$.

Для завершения решения задачи ЛП (3.22)-(3.23) нужно установить соответствие между каждой из оптимальных стратегий торговли и стоимостью соответствующего портфеля в момент $k=0$ (или, другими словами, с соответствующим значением верхней справедливой цены $\bar{x}(0))$. Эти значения, с другой стороны, являются оптимальными значениями целевого функционала (3.22). Подставим каждый из приведенных выше триплетов $\sigma^{--}$, $\sigma^{++}, \sigma^{+-}$и $\sigma^{-+}$в (3.22). Нетрудно проверить, что начальная стоимость портфеля, определяемого стратегией торговли $\sigma^{--}$, равна $x_{--}$(см. (3.18)), далее, начальные стоимости портфеля $x_{++}, x_{+-}$и $x_{-+}$(см. (3.15), (3.16) и (3.17)) отвечают, соответственно, стратегиям торговли $\sigma^{++}, \sigma^{+-}$и $\sigma^{-+}$. Напомним, что значения $x_{--}, x_{++}, x_{+-}$и $x_{-+}$совпадают также с оптимальным значением целевого функционала дуальной задачи ЛП (3.20)-(3.21).

Зная, что соответствующие оптимальные значения целевых функционалов в дуальных задачах ЛП совпадают друг с другом, мы приходим к следуюшей теореме, которая подводит итог приведенному выше обсуждению.

Теорема 1. Пусть $X=f\left(s^{(1)}\right)-$ платежное обязательство. и пусть множества $S_{+}$, $S_{-}$и $S_{0}$ определяются согласно (3.9) для функции выплат $f$.

(а) Если $s^{(0)} \in S_{-}$или $s^{(0)} \in S_{+}$, то верхние справеоливые цены $\bar{x}(0)$ платежного обязательства $X$, а также соответствующие верхние хеджирующие стратегии можно записать в виде таблии 1 и 2. Каждая клетка таблицы содержит пару (верхняя справедливая иена; верхняя хеджирующая стратегия).

(б) Если $s^{(0)} \in S_{0}$, то верхняя справедливая чена платежного обязательства $X=f\left(s^{(1)}\right)$ в момент $k=0$ существует, единственна и равна

$$
x_{++}=x_{+-}=x_{-+}=x_{--} .
$$


Таблица 2.

\begin{tabular}{|c|c|c|c|}
\hline & $p_{1}<p_{2}$ & $p_{1}>p_{2}$ & $p_{1}=p_{2}=p$ \\
\hline$s^{(0)} \in S_{+}$ & $\left(x_{+-} ; \sigma^{+-}\right)$ & $\left(x_{-+} ; \sigma^{-+}\right)$ & $\left(x_{+-}=x_{-+} ; \sigma=\alpha \sigma^{+-}+(1-\alpha) \sigma_{-+}\right)$ \\
& & & $0 \leqslant \alpha \leqslant 1$ \\
\hline
\end{tabular}

Соответствующая единственная верхняя хеджирующая стратегия равна

$$
\sigma^{++}=\sigma^{+-}=\sigma^{-+}=\sigma^{--} \text {. }
$$

Доказательство. Часть (б) проверяется непосредственными вычислениями.

Остается рассмотреть случай $s^{(0)} \in S_{-}$и $p_{1}+p_{2}=1$, а также случай $s^{(0)} \in S_{+}$и $p_{1}=p_{2}=p$. Все оставшиеся случаи были рассмотрены выше.

Пусть $s^{(0)} \in S_{-}$, или, что эквивалентно, $d_{f}<0$. Имеются две допустимые торговые стратегии $\sigma^{--}$и $\sigma^{++}$. Если $p_{1}+p_{2}=1$, то соответствующие стоимости портфелей $x_{--}$и $x_{++}$совпадают друг с другом (см. (3.18) и (3.15)). Обе стратегии торговли $\sigma^{--}$ и $\sigma^{++}$по построению являются вершинами допустимой области. Отсюда вытекает, что любая стратегия $\sigma$, которая задается выпуклой комбинацией стратегий $\sigma^{--}$и $\sigma^{++}$, является оптимальным решение задачи (3.22)-(3.23), отвечающим оптимальному значению $x_{--} \equiv x_{++}$целевого функционала.

Случай $s^{(0)} \in S_{+}$и $p_{1}=p_{2}=p$ рассматривается аналогично.

\section{4. Нижние справедливая цена и хеджирующие стратегии}

Нижняя справедливая цена платежного обязательства - это максимальная цена, которую покупатель опциона готов заплатить, с сохранением надежды на то, что выплаты в момент исполнения покроют расходы на покупку. С точки зрения продавца опциона, это минимальный капитал, который позволит инвестору выполнить обязательства по контракту с положительной вероятностью. Обозначим через $\underline{x}(0)$ нижнюю справедливую цену платежного обязательства $X=f\left(s^{(1)}\right)$. Тогда $\underline{x}(0)$ - это такое минимальное значение начального капитала, что для соответствующего значения капитала в момент испилнения выполняется неравенство

$$
x(1) \leqslant f\left(s^{(1)}\right)
$$

(ср. с (3.1)). С другой стороны, известно, что

$$
\underline{x}(0)=\rho^{-1} \inf _{Q \in 2} \mathbf{E}_{Q} f\left(s_{01} \xi_{1}, s_{02} \xi_{2}\right)
$$

(см., например, утверждение 1.28 в [7]). Следуя используемому в предыдущих разделах подходу, основанному на линейном программировании, сформулируем две взаимно дуальные задачи ЛП, отвечающие случаю нижней справедливой цены и соответствующей нижней хеджирующей стратегии (или просто нижнему хеджу).

Задача ЛП 1.

$$
\text { минимизировать } Y_{1} f_{++}+Y_{2} f_{+-}+Y_{3} f_{-+}+Y_{4} f_{--}
$$


Таблица 3.

\begin{tabular}{|c|c|c|c|}
\hline & $p_{1}<p_{2}$ & $p_{1}>p_{2}$ & $p_{1}=p_{2}=p$ \\
\hline$s^{(0)} \in S_{-}$ & $\left(x_{+-} ; \sigma^{+-}\right)$ & $\left(x_{-+}: \sigma^{-+}\right)$ & $\left(x_{+}=x_{-+}: \sigma=\alpha \sigma^{+-}+(1-\alpha) \sigma_{-+}\right)$ \\
& & & $0 \leqslant \alpha \leqslant 1$ \\
\hline
\end{tabular}

Таблица 4.

\begin{tabular}{|c|c|c|c|}
\hline & $p_{1}+p_{2}<1$ & $p_{1}+p_{2}>1$ & $p_{1}+p_{2}=1$ \\
\hline$s^{(0)} \in S_{+}$ & $\left(x_{++} ; \sigma^{++}\right)$ & $\left(x_{--i} \sigma^{--}\right)$ & $\left(x_{++}=x_{--} ; \sigma=\alpha \sigma^{++}+(1-\alpha) \sigma_{--}\right)$ \\
& & & $0 \leqslant \alpha \leqslant 1$ \\
\hline
\end{tabular}

при условин

$$
\begin{aligned}
u_{1}\left(Y_{1}+Y_{2}\right)+d_{1}\left(Y_{3}+Y_{4}\right) & =1, \\
u_{2}\left(Y_{1}+Y_{3}\right)+d_{2}\left(Y_{2}+Y_{4}\right) & =1, \\
Y_{1}+Y_{2}+Y_{3}+Y_{4} & =1 / \rho, \\
Y_{1}, Y_{2}, Y_{3}, Y_{4} & \geqslant 0 .
\end{aligned}
$$

Задача ЛП 2.

$$
\text { максимизировать } \beta b_{0}+\gamma_{1} s_{0,1}+\gamma_{2} s_{0,2}
$$

при условии

$$
\begin{aligned}
& \beta b_{0} \rho+\gamma_{1} s_{0,1} u_{1}+\gamma_{2} s_{0,2} u_{2} \leqslant f_{++} \\
& \beta b_{0} \rho+\gamma_{1} s_{0,1} u_{1}+\gamma_{2} s_{0,2} d_{2} \leqslant f_{+-} \\
& \beta b_{0} \rho+\gamma_{1} s_{0,1} d_{1}+\gamma_{2} s_{0,2} u_{2} \leqslant f_{-+} \\
& \beta b_{0} \rho+\gamma_{1} s_{0,1} d_{1}+\gamma_{2} s_{0,2} d_{2} \leqslant f_{--}
\end{aligned}
$$

При $Y_{j}=q_{j} / \rho$ целевая функция (3.35) превращается в (3.34), а ограничения на допустимую область (3.36) преврашаются в (3.4).

Целевая функция (3.37) отвечает стоимости портфеля в момент $k=0$, а ограничения, определяющие допустимую область (3.38), отвечают ограничениям (3.33).

Используя методику, аналогичную методике из предыдущих разделов, приходим к следующей теореме.

Теорема 2. Пусть $X=f\left(s^{(1)}\right)$ - платежное обязательство, и пусть множества $S_{+}$, $S_{-}$и $S_{0}$ определены в (3.9) для платежной функции $f$.

(а) $E с л и s^{(0)} \in S_{-}$или $s^{(0)} \in S_{+}$, то нижняя справедливая иена $\underline{x}(0)$ обязательства $X$ и соответствующая нижняя хеджирующая стратегия задаются табличами 3 и 4. Каждая клетка таблищы содержит пару (нижняя справедливая чена; нижняя хеджирующая стратегия).

(б) Если $s^{(0)} \in S_{0}$, то существует единственная нижняя справедливая чена платежного обязательства $X=f\left(s^{(1)}\right)$ в момент $k=0$

$$
x_{++}=x_{+-}=x_{-+}=x_{--} .
$$


Соответствующая нижняя хеджирующая стратегия равна

$$
\sigma^{++}=\sigma^{+-}=\sigma^{-+}=\sigma^{--} \text {. }
$$

Следствие 1. Из теоремы 1, часть (б), и теоремы 2, часть (б), очевидно, следует, что в случае $s^{(0)} \in S_{0}$ платежное обязательство $X=f\left(s^{(1)}\right)$ достижимо при использовании единственной стратегии торговли

$$
\sigma^{++}=\sigma^{+-}=\sigma^{-+}=\sigma^{--} .
$$

Единственная справедливая цена обязательства $X$ в момент $k=0$ равна

$$
x_{++}=x_{+-}=x_{-+}=x_{--} .
$$

\section{5. Арбитражные возможности на одношаговом рынке}

Для каждого случая $s^{(0)} \in S_{+}$и $s^{(0)} \in S_{-}$при любом соотношении между $p_{1}$ и $p_{2}$ мы нашли интервал справедливых цен $(\underline{x}(0), \bar{x}(0))$. Подчеркнем, что этот интервал является открытым. Его границы являются точками арбитража. Это следует, например, из утверждения 1.16 в [7], которое представляет собой версию так называемой фундаментальной теоремы оценивания опционов.

Теорема 3 ([7]). Арбитражных возможностей не существует тогда и только тогда, когда существует нейтральная к риску вероятностная мера.

Как указано выше, мартингальные меры $Q_{\lambda_{+}}$и $Q_{\lambda_{-}}$, участвующие в подсчете величин $\underline{x}(0)$ и $\bar{x}(0)$, не принадлежат множеству мер, нейтральных к риску в смысле определения 1 .

Рассмотрим конкретную арбитражную ситуацию. Пусть, для примера, $s^{(0)} \in S_{+}$и $p_{1}<p_{2}$. Тогда по теореме 1 верхняя справедливая цена платежного обязательства $X$ в момент $k=0$ равна $x_{+-}$, а соответствующая хеджирующая стратегия равна $\sigma^{+-}$. Если инвестор продает платежное обязательство $X$ по цена $x_{+-}$в момент $k=0$, a затем использует стратегию $\sigma^{+-}$, то в момент исполнения обязательства $k=1$ цена его портфеля будет

$$
x(1)=\beta^{+-} b_{0} \rho+\gamma_{1}^{+-} s_{0,1} \xi_{1}+\gamma_{2}^{+-} s_{0,2} \xi_{2} .
$$

По построению, если вектор скачков $\xi=\left(\xi_{1}, \xi_{2}\right)$ принимает значения $\left(u_{1}, u_{2}\right),\left(d_{1}, u_{2}\right)$ или $\left(d_{1}, d_{2}\right)$, то выполняется равенство $x(1)=f\left(s^{(1)}\right)$; если вектор скачков принимает значение $\left(u_{1}, d_{2}\right)$, то

$$
\bar{\delta}_{1}=x(1)-f\left(s^{(1)}\right)=d_{f}>0
$$

и инвестор фиксирует положительный безрисковый доход $\bar{\delta}_{1}$. Легко проверить, что во всех других случаях, за исключением случая $s^{(0)} \in S_{0}$, имеет место та же самая ситуация: величина $\bar{\delta}_{1}$ равна нулю, если вектор скачков принимает три значения из четырех, а в четвертом случае инвестор получает безрисковый доход

$$
\bar{\delta}_{1}=x(1)-f\left(s^{(1)}\right)=\left|d_{f}\right|
$$

который реализует арбитражную возможность для продавца платежного обязательства. 
Если инвестор продает платежное обязательство $X$ в момент $k=0$ по нижней справедливой цене и затем использует соответствующую нижнюю хеджирующую стратегию, то мы получаем обратную ситуацию. Если вектор скачков принимает одно соответствующее значение из четырех возможных, то инвестор несет потери в размере

$$
\underline{\delta}_{1}=f\left(s^{(1)}\right)-x(1)=\left|d_{f}\right| .
$$

Это создает арбитражную возможность для покупателя платежного обязательства $X$. В самом деле, предположим, что покупатель приобретает платежное обязательство $X$ в момент $k=0$ по нижней справедливой цене, а для финансирования покупки продает нижний хеджирующий портфель. Если вектор скачков принимает одно соответствующее значение из четырех возможных, то покупатель обязательства получает доход величины (3.41).

\section{4. Динамический двумерный бинарный рынок}

В этом разделе мы распространим результаты предыдущих разделов на случай динамического рынка. Напомним, что мы рассматриваем европейское платежное обязательство $X$ с платежной функцией $f$. Мы предполагаем, что исходным моментом является $k=0$, а дата исполнения обязательства равна $k=n$, так что $X=f\left(s^{(n)}\right)$, где $s^{(k)}=\left(s_{k, 1}, s_{k, 2}\right)$, $k=0,1, \ldots, n,-$ вектор цен акций в момент $k$. Обозначим

$$
\xi^{(k)}=\left(\xi_{k, 1}, \xi_{k, 2}\right)=\left(\frac{s_{k, 1}}{s_{k-1,1}}, \frac{s_{k, 2}}{s_{k-1,2}}\right)
$$

вектор скачков последовательных цен акций в момент $k$.

\section{1. Обозначения и предварительные соображения}

Введем некоторые обозначения и определим некоторые важные объекты. Вначале определим необходимое для дальнейших рассуждений выборочное пространство $\Omega^{(k)}$, $k=1, \ldots, n$, которое состоит из $N_{k}=4^{k}$ элементов: $\Omega^{(k)}=\left\{\omega_{1}^{(k)}, \omega_{2}^{(k)}, \ldots, \omega_{N_{k}}^{(k)}\right\}$. Здесь каждый элемент $\omega_{i}^{(k)}$ - это возможное состояние природы, которое неизвестно в момент 0 и становится известным в момент $k$. Каждое состояние $\omega_{i}^{(k)}, i=1, \ldots, N_{k},-$ это $k$ возможных последовательных значений вектора скачков $\xi^{(r)}=\left(\xi_{r, 1}, \xi_{r, 2}\right), r=1, \ldots, k$. Для упрощения последующих обсуждений всюду далее мы будем рассматривать эквивалентное выборочное пространство $\widetilde{\Omega}^{(k)}=\left\{\widetilde{\omega}_{1}^{(k)}, \widetilde{\omega}_{2}^{(k)}, \ldots, \widetilde{\omega}_{N_{k}}^{(k)}\right\}$. Здесь каждому состоянию $\widetilde{\omega}_{i}^{(k)}$ отвечает точка в пространстве $(\{0,1\} \times\{0,1\})^{k}$. Например, выборочное пространство $\tilde{\Omega}^{(1)}=\left\{\tilde{\omega}_{1}^{(1)}, \tilde{\omega}_{2}^{(1)}, \tilde{\omega}_{3}^{(1)}, \tilde{\omega}_{4}^{(1)}\right\}$ состоит из точек

$$
\tilde{\omega}_{1}^{(1)}=(1,1), \quad \tilde{\omega}_{2}^{(1)}=(1,0), \quad \tilde{\omega}_{3}^{(1)}=(0,1), \quad \tilde{\omega}_{4}^{(1)}=(0,0) .
$$

Очевидно, что существует взаимно однозначное соответствие между выборочными пространствами $\Omega^{(1)}$ и $\widetilde{\Omega}^{(1)}$, и любую вероятностную меру $Q$, определенную на $\Omega^{(1)}$, можно перенести на $\widetilde{\Omega}^{(1)}$ следующим естественным образом: $Q\left(\omega_{k}^{(1)}\right)=Q\left(\tilde{\omega}_{k}^{(1)}\right), k=1,2,3,4$.

Пусть $Q$ - мера на выборочном пространстве одношагового рынка $\widetilde{\Omega}^{(1)}$

$$
Q\left(\tilde{\omega}_{l}^{(1)}\right)=q_{l}, \quad q_{l} \geqslant 0, \quad l=1,2,3,4, \quad q_{1}+q_{2}+q_{3}+q_{4}=1 .
$$


Предположим, что векторы скачков $\xi^{(k)}=\left(\xi_{k, 1}, \xi_{k, 2}\right), k=1, \ldots, n$, являются независимыми случайными величинами на выборочном пространстве $\left(\tilde{\Omega}^{(1)}, Q\right)$, имеющими одно и то же распределение $Q$. Достаточно просто определить соответствующую меру $Q^{(k)}$ на выборочном пространстве $\widetilde{\Omega}^{(k)}$, положив

$$
Q^{(k)}\left(\tilde{\omega}^{(k)}\right)=q_{1}^{j_{1}} q_{2}^{j_{2}} q_{3}^{j_{3}} q_{4}^{j_{4}},
$$

где $\mathbf{j}=\left(j_{1}, j_{2}, j_{3}, j_{4}\right) \in J_{k}$,

$$
J_{k}=\left\{\mathbf{j}: \mathbf{j}=\left(j_{1}, j_{2}, j_{3}, j_{4}\right), j_{l} \geqslant 0, l=1,2,3,4, j_{1}+j_{2}+j_{3}+j_{4}=k\right\} .
$$

Здесь $j_{l}, l=1,2,3,4,-$ число появлений значения вектора скачков $\tilde{\omega}_{l}^{(1)}$ в наборе $\tilde{\omega}^{(k)}$. Если обозначить через $\widetilde{\Omega}_{\mathrm{j}}^{(k)}$ подмножество множества $\widetilde{\Omega}^{(k)}$, состоящее из состояний $\widetilde{\omega}^{(k)}$, для которых вектор ј принимает заданное значение $\left(j_{1}, j_{2}, j_{3}, j_{4}\right)$ (то есть имеется $j_{1}$ появлений $\widetilde{\omega}_{1}^{(1)}, j_{2}$ появлений $\widetilde{\omega}_{2}^{(1)}, j_{3}$ появлений $\widetilde{\omega}_{3}^{(1)}$ и $j_{4}$ появлений $\widetilde{\omega}_{4}^{(1)}$ для каждого $\left.\widetilde{\omega}^{(k)} \in \widetilde{\Omega}_{\mathrm{j}}^{(k)}\right)$, то $Q^{(k)}\left(\widetilde{\Omega}_{\mathrm{j}}^{(k)}\right)$ задается полиномиальным вероятностным распределением

$$
Q^{(k)}\left(\widetilde{\Omega}_{\mathrm{j}}^{(k)}\right)=b_{k}(\mathbf{j}, \mathbf{q})=\frac{k !}{j_{1} ! j_{2} ! j_{3} ! j_{4} !} q_{1}^{j_{1}} q_{2}^{j_{2}} q_{3}^{J_{3}} q_{4}^{j_{4}},
$$

где $\mathbf{q}=\left(q_{1}, q_{2}, q_{3}, q_{4}\right), q_{l} \geqslant 0, l=1,2,3,4, q_{1}+q_{2}+q_{3}+q_{4}=1$.

Определение 2. Вероятностная мера $Q^{(n)}$ называется нейтральной к риску (или эквивалентной мартингальной мерой) для динамического рынка с горизонтом $n$, если

(a) $Q^{(n)}\left(\widetilde{\omega}^{(n)}\right)>0$ для каждого элемента $\widetilde{\omega}^{(n)} \in \widetilde{\Omega}^{(n)}$, и

(б) дисконтированный вектор цен является мартингалом относительно $Q^{(n)}$, то есть

$$
\mathbf{E}_{Q^{(n)}}\left(s^{*(n)} \mid \mathscr{F}_{k}\right)=s^{*(k)}, \quad k=0,1, \ldots, n,
$$

где $\left\{\mathscr{F}_{k}, k=0,1, \ldots, n\right\}=\mathbf{F}-$ фильтрация, порожденная процессом цен акций $s^{(l)}$ $(l=0,1, \ldots, n)$.

Из свойств условных математических ожиданий легко следует, что $Q^{(n)}$ является мартингальной мерой на рынке с горизонтом $n$, если $Q$ является мартингальной мерой на соответствующем одношаговом рынке. Очевидно также (см., например, (4.2)), что $Q^{(n)}$ удовлетворяет части (а) определения 2 (то есть является эквивалентной мерой), если $Q$ удовлетворяет части (а) определения 1.

Рассмотрим набор функций

$$
f^{(k)}(s)=\rho^{-(n-k)} \sum_{j \in J_{n-k}} b_{n-k}(\mathbf{j}, \mathbf{q}) f\left(s_{1} u_{1}^{j_{1}+j_{2}} d_{1}^{j_{3}+j_{4}}, s_{2} u_{2}^{j_{1}+J_{3}} d_{2}^{l_{2}+j_{4}}\right),
$$

где $k=0,1, \ldots, n-1, s=\left(s_{1}, s_{2}\right) \in \mathbf{R}_{+}^{2}$, и $f-$ платежная функция европейского финансового обязательства $X$. Заметим, что для $k=n$ справедливо равенство $f^{(n)}(s)=f(s)$. Легко проверить, что

$$
\begin{aligned}
f^{(k)}(s)=\rho^{-1}\left(f^{(k+1)}\left(s_{1} u_{1}, s_{2} u_{2}\right)\right. & q_{1}+f^{(k+1)}\left(s_{1} u_{1}, s_{2} d_{2}\right) q_{2} \\
& \left.+f^{(k+1)}\left(s_{1} d_{1}, s_{2} u_{2}\right) q_{3}+f^{(k+1)}\left(s_{1} d_{1}, s_{2} d_{2}\right) q_{4}\right)
\end{aligned}
$$


для $k=0,1, \ldots, n-1$. Введем обозначения

$$
\begin{array}{ll}
f_{++}^{(k)}(s)=f^{(k+1)}\left(s_{1} u_{1}, s_{2} u_{2}\right), & f_{+-}^{(k)}(s)=f^{(k+1)}\left(s_{1} u_{1}, s_{2} d_{2}\right), \\
f_{-+}^{(k)}(s)=f^{(k+1)}\left(s_{1} d_{1}, s_{2} u_{2}\right), & f_{--}^{(k)}(s)=f^{(k+1)}\left(s_{1} d_{1}, s_{2} d_{2}\right) .
\end{array}
$$

Положим теперь $s=s^{(k)}, k=0,1, \ldots, n-1$, где $s^{(k)}-$ вектор цен акщий в момент $k$. Для краткости будем использовать упрощенные обозначения

$$
f_{++}^{(k)}=f_{++}^{(k)}\left(s^{(k)}\right), \quad f_{+-}^{(k)}=f_{+-}^{(k)}\left(s^{(k)}\right), \quad f_{-+}^{(k)}=f_{-+}^{(k)}\left(s^{(k)}\right), \quad f_{--}^{(k)}=f_{--}^{(k)}\left(s^{(k)}\right) .
$$

Для $s=s^{(k)}, k=0,1, \ldots, n-1$, с использованием всех приведенных выше обозначений соотношение (4.5) принимает вид

$$
f^{(k)}\left(s^{(k)}\right)=\rho^{-1}\left(f_{++}^{(k)} q_{1}+f_{+-}^{(k)} q_{2}+f_{-+}^{(k)} q_{3}+f_{--}^{(k)} q_{4}\right) .
$$

Замечание 4. Представление (4.4) эквивалентно представлению

$$
f^{(k)}\left(s^{(k)}\right)=\rho^{-(n-k)} \mathbf{E}_{Q^{(n-k)}}\left(f\left(s^{(n)}\right)\right)
$$

для $s=s^{(k)}, k=0,1, \ldots, n-1$.

Определение 3. Пусть $f-$ функция, заданная на $\mathbf{R}_{+}^{2}$, и пусть множества $S_{+}$и $S_{-}$определены согласно (3.9). Будем говорить, что $f$ положительно определена на $\mathbf{R}_{+}^{2}$, если для любого $s \in \mathbf{R}_{+}^{2}$ справедливо неравенство $d_{f}(s) \geqslant 0$ и множество $S_{+}$не пусто. Аналогично, $f$ отрицательно определена на $\mathbf{R}_{+}^{2}$, если для любого $s \in \mathbf{R}_{+}^{2}$ справедливо неравенство $d_{f}(s) \leqslant 0$ и множество $S_{-}$не пусто.

Например, положительно определена платежная функция европейского пакетного опциона покупки (см. пример в разделе 3.2 ).

Предложение 2. Если функция $f=f^{(n)}$ положительно (отрицательно) определена, то этим же свойством обладает функиия $f^{(k)}$ при любом $k=0,1, \ldots, n-1$. Если $d_{f}(s)=0$ для любого $s \in \mathbf{R}_{+}^{2}$, или, другими словами, $S_{0}=\mathbf{R}_{+}^{2}$, то $d_{f^{(k)}}(s)=0$ для любого $s \in \mathbf{R}_{+}^{2}$, $k=0,1, \ldots, n-1$.

Доказательство. Легко проверить, что для любого $s \in \mathbf{R}_{+}^{2}$

$$
d_{f^{(n-1)}}(s)=\rho^{-1} \sum_{k=1}^{4} q_{k} d_{g_{k}}(s),
$$

где

$$
\begin{array}{ll}
g_{1}(s)=f\left(s_{1} u_{1}, s_{2} u_{2}\right), & g_{2}(s)=f\left(s_{1} u_{1}, s_{2} d_{2}\right), \\
g_{3}(s)=f\left(s_{1} d_{1}, s_{2} u_{2}\right), & g_{4}(s)=f\left(s_{1} d_{1}, s_{2} d_{2}\right),
\end{array}
$$

а $q_{k}$ - неотрицательные константы, фигурирующие в (4.5). Если функция $f$ положительно (отрицательно) определена, то этим же свойством обладают четыре функции $g_{k}$, $k=1, \ldots, 4$. Из соотношения (4.9) легко получается, что в этом случае функция $f^{(n-1)}$ также положительно (отрицательно) определена. Если $d_{f}(s)=0$ для любого $s \in \mathbf{R}_{+}^{2}$, то $d_{g_{k}}(s)=0$ для любого $s \in \mathbf{R}_{+}^{2}$, и из (4.9) получаем, что $d_{f(n-1)}(s)=0$. Итерация этих рассуждений для $k=n-2, n-3, \ldots, 0$ завершает доказательство. 
Перейдем к следующему набору обозначений. Определим четыре триплета $\sigma^{+-}(k)=\left(\beta_{k}^{+-}, \gamma_{k, 1}^{+-}, \gamma_{k, 2}^{+-}\right)$где

$$
\gamma_{k, 1}^{+-}=\frac{f_{++}^{(k)}-f_{-+}^{(k)}}{s_{k, 1}\left(u_{1}-d_{1}\right)}, \quad \gamma_{k, 2}^{+-}=\frac{f_{-+}^{(k)}-f_{--}^{(k)}}{s_{k, 2}\left(u_{2}-d_{2}\right)}
$$

и

$$
\beta_{k}^{+-}=\frac{1}{b_{k} \rho}\left(-\frac{d_{1}}{u_{1}-d_{1}} f_{++}^{(k)}+\left(\frac{d_{1}}{u_{1}-d_{1}}-\frac{d_{2}}{u_{2}-d_{2}}\right) f_{-+}^{(k)}+\frac{u_{2}}{u_{2}-d_{2}} f_{--}^{(k)}\right)
$$

$\sigma^{-+}(k)=\left(\beta_{k}^{-+}, \gamma_{k, 1}^{-+}, \gamma_{k, 2}^{-+}\right)$где

$$
\gamma_{k, 1}^{-+}=\frac{f_{+-}^{(k)}-f_{--}^{(k)}}{s_{k, 1}\left(u_{1}-d_{1}\right)}, \quad \gamma_{k, 2}^{-+}=\frac{f_{++}^{(k)}-f_{+-}^{(k)}}{s_{k, 2}\left(u_{2}-d_{2}\right)}
$$

и

$$
\beta_{k}^{-+}=\frac{1}{b_{k} \rho}\left(-\frac{d_{2}}{u_{2}-d_{2}} f_{++}^{(k)}+\left(\frac{d_{2}}{u_{2}-d_{2}}-\frac{d_{1}}{u_{1}-d_{1}}\right) f_{+-}^{(k)}+\frac{u_{1}}{u_{1}-d_{1}} f_{--}^{(k)}\right)
$$

$\sigma^{++}(k)=\left(\beta_{k}^{++}, \gamma_{k, 1}^{++}, \gamma_{k, 2}^{++}\right)$где

$$
\gamma_{k, 1}^{++}=\frac{f_{+-}^{(k)}-f_{--}^{(k)}}{s_{k, 1}\left(u_{1}-d_{1}\right)}, \quad \gamma_{k, 2}^{++}=\frac{f_{-+}^{(k)}-f_{--}^{(k)}}{s_{k, 2}\left(u_{2}-d_{2}\right)}
$$

и

$$
\beta_{k}^{++}=\frac{1}{b_{k} \rho}\left(-\frac{d_{1}}{u_{1}-d_{1}} f_{+-}^{(k)}-\frac{d_{2}}{u_{2}-d_{2}} f_{-+}^{(k)}+\left(1+\frac{d_{1}}{u_{1}-d_{1}}+\frac{d_{2}}{u_{2}-d_{2}}\right) f_{--}^{(k)}\right) ;
$$

$\sigma^{--}(k)=\left(\beta_{k}^{--}, \gamma_{k, 1}^{--}, \gamma_{k, 2}^{--}\right)$где

$$
\gamma_{k, 1}^{--}=\frac{f_{++}^{(k)}-f_{-+}^{(k)}}{s_{k, 1}\left(u_{1}-d_{1}\right)}, \quad \gamma_{k, 2}^{--}=\frac{f_{++}^{(k)}-f_{+-}^{(k)}}{s_{k, 2}\left(u_{2}-d_{2}\right)}
$$

и

$$
\beta_{k}^{--}=\frac{1}{b_{k} \rho}\left(\left(1-\frac{u_{1}}{u_{1}-d_{1}}-\frac{u_{2}}{u_{2}-d_{2}}\right) f_{++}^{(k)}+\frac{u_{2}}{u_{2}-d_{2}} f_{+-}^{(k)}+\frac{u_{1}}{u_{1}-d_{1}} f_{-+}^{(k)}\right) .
$$

\section{2. Верхняя и нижняя справедливые цены, верхний и нижний хеджи}

Теорема 4. Пусть $X=f\left(s^{(n)}\right)$ - европейское платежсное обязательство на двумерном бинарном рынке с горизонтом $n$.

(a) Пусть платежная функция $f$ положительно или отрицательно определена на $\mathbf{R}_{+}^{2}$. Тогда верхняя справедливая иена платежного обязательства $X$ в момент $k$ равна

$$
\bar{x}(k)=f^{(k)}\left(s^{(k)}\right), \quad k=0,1, \ldots, n-1 .
$$


Таблица 5.

\begin{tabular}{|c|c|c|c|}
\hline & $p_{1}<p_{2}$ & $p_{1}>p_{2}$ & $p_{1}=p_{2}=p$ \\
\hline $\begin{array}{c}f \text { положительно } \\
\text { определена }\end{array}$ & $\left(Q_{\lambda_{+}} ; \sigma^{+-}(k)\right)$ & $\left(Q_{\lambda_{+}} ; \sigma^{-+}(k)\right)$ & $\begin{array}{c}\left(Q_{\lambda_{+}} ; \alpha \sigma^{+-}(k)+(1-\alpha) \sigma^{-+}(k)\right) \\
0 \leqslant \alpha \leqslant 1\end{array}$ \\
\hline
\end{tabular}

Таблица 6.

\begin{tabular}{|c|c|c|c|}
\hline & $p_{1}+p_{2}<1$ & $p_{1}+p_{2}>1$ & $p_{1}+p_{2}=1$ \\
\hline $\begin{array}{c}f \text { отрицательно } \\
\text { определена }\end{array}$ & $\left(Q_{\lambda_{-}} ; \sigma^{++}(k)\right)$ & $\left(Q_{\lambda_{-}} ; \sigma^{--}(k)\right)$ & $\left(Q_{\lambda_{-}} ; \alpha \sigma^{++}(k)+(1-\alpha) \sigma^{--}(k)\right)$ \\
& & $0 \leqslant \alpha \leqslant 1$ \\
\hline
\end{tabular}

Здесь $f^{(k)}$ определяется согласно (4.4) с параметрами $q_{l}, l=1,2,3,4$, которые являются компонентами верхней или нижней мартингальной меры: $Q_{\lambda_{+}}$или $Q_{\lambda_{-}}$ (см. замечание 1). Соответствуючий выбор мартингальной меры определяется табличами 5 и 6. Каждая верхняя справедливая чена определяет соответствуюцую верхнюю хеджирующую стратегию $\bar{\sigma}(k)=\left(\bar{\beta}_{k}, \bar{\gamma}_{k, 1}, \bar{\gamma}_{k, 2}\right)$, которая выбирается в соответствии с табличами 5 и 6. Каждая клетка таблии 5 и 6 содержит пару (верхняя/нижняя мартингальная мера; верхняя хеджирующая стратегия) для каждой возможной ситуачии.

(б) Если платежная функция $f$ такова, что $d_{f}(s)=0$ для любого $s \in \mathbf{R}_{+}^{2}$, то соотночение (4.10) определяет единственную верхнюю справедливую чену платежного обязательства $X$ в момент $k, k=0,1, \ldots, n-1$, и результат не зависит от выбора мартингальной меры $Q_{\lambda_{+}}$или $Q_{\lambda_{-}}$. Соответствующая единственная хеджируюшая стратегия в момент $k$ равна

$$
\sigma^{++}(k)=\sigma^{+-}(k)=\sigma^{-+}(k)=\sigma^{--}(k) .
$$

Доказательство. Докажем часть (а).

Мы докажем утверждение теоремы только для одного случая, содержащегося в таблицах 5 и 6. Остальные случаи рассматриваются аналогично.

Предположим, что платежная функция $f$ положительно определена, и пусть $p_{1}<p_{2}$. Положим $s^{(0)}=s^{(n-1)}$ и $s^{(1)}=s^{(n)}$. Тем самым мы сведем ситуацию к одношаговому рынку, рассмотренному в предыдущих разделах. Применим часть (а) теоремы 1 с выбором обозначений, соответствующих рассматриваемому случаю. Из части (а) теоремы 1 следует, что верхняя справедливая цена платежного обязательства $X$ в момент $k=n-1$ задается равенством

$$
\bar{x}(n-1)=\rho^{-1}\left(f_{++}^{(n-1)} p_{1}+f_{-+}^{(n-1)}\left(p_{2}-p_{1}\right)+f_{--}^{(n-1)}\left(1-p_{2}\right)\right)
$$

или, с учетом (4.7),

$$
\bar{x}(n-1)=f^{(n-1)}\left(s^{(n-1)}\right),
$$

где $\left(q_{1}, q_{2}, q_{3}, q_{4}\right)=\left(p_{1}, 0, p_{2}-p_{1}, 1-p_{2}\right)$ являются компонентами верхней мартингальной меры $Q_{\lambda_{+}}$, выбранной для случая $p_{1}<p_{2}$ (см. замечание 1). Соответствующий верхний хедж определяется равенством

$$
\bar{\sigma}(n-1)=\left(\bar{\beta}_{n-1}, \bar{\gamma}_{n-1,1}, \bar{\gamma}_{n-1,2}\right),
$$


Таблица 7.

\begin{tabular}{|c|c|c|c|}
\hline & $p_{1}<p_{2}$ & $p_{1}>p_{2}$ & $p_{1}=p_{2}=p$ \\
\hline $\begin{array}{c}f \text { отрицательно } \\
\text { определена }\end{array}$ & $\left(Q_{\lambda_{+}} ; \sigma^{+-}(k)\right)$ & $\left(Q_{\lambda_{+}} ; \sigma^{-+}(k)\right)$ & $\left(Q_{\lambda_{+}} ; \alpha \sigma^{+-}(k)+(1-\alpha) \sigma^{-+}(k)\right)$ \\
$0 \leqslant \alpha \leqslant 1$
\end{tabular}

где

$$
\bar{\gamma}_{n-1,1}=\gamma_{n-1,1}^{+-}, \quad \bar{\gamma}_{n-1,2}=\gamma_{n-1,2}^{+-}, \quad \bar{\beta}_{n-1}=\beta_{n-1}^{+-}
$$

(формулы для $\gamma_{k, 1}^{+-}, \gamma_{k, 2}^{+-}$и $\beta_{k}^{+-}, k=0, \ldots, n-1$, приведены в разделе 4.1). Заметим, что приведенные представления для $\bar{x}(n-1)$ и $\bar{\sigma}(n-1)$ справедливы вне зависимости от того, какой из случаев $s^{(n-1)} \in S_{+}$или $s^{(n-1)} \in S_{0}$ имеет место (здесь множества $S_{+}$и $S_{0}$ соответствуют функции $\left.f=f^{(n)}\right)$.

Выберем теперь $f^{(n-1)}$ в качестве новой платежной функции платежного обязательства $X$ на новом одношаговом рынке с начальной точкой $k=n-2$ и датой исполнения $k=n-1$. Поскольку функция $f^{(n-1)}$ наследует от функции $f$ свойство положительной определенности (см. предложение 2), мы можем повторить приведенные выше рассуждения для этой новой задачи. Действуя таким образом, мы дойдем до случая $k=0$ и получим искомую формулу, что завершает доказательство.

Справедливость части (б) легко вытекает из части (б) теоремы 1 с использованием абсолютно аналогичных рассуждений.

Сформулируем теперь аналогичное утверждение, описывающее нижние справедливые цены и нижние хеджирующие стратегии для двумерного бинарного рынка с горизонтом $n$. Мы опускаем доказательство следующей теоремы, так как оно аналогично доказательству вышеприведенной теоремы 4.

Теорема 5. Пусть $X=f\left(s^{(n)}\right)$ - европейское платежное обязательство на двумерном бинарном рынке с горизонтом $п$.

(a) Пусть платежная функиия $f$ положительно или отрицательно определена на $\mathbf{R}_{+}^{2}$. Тогда нижняя справедливая цена платежного обязательства Х в момент $k$ равна

$$
\underline{x}(k)=f^{(k)}\left(s^{(k)}\right), \quad k=0,1, \ldots, n-1 .
$$

Здесь $f^{(k)}$ определяется согласно (4.4) с параметрами $q_{l}, l=1,2,3,4$, которые являются компонентами верхней или нижней мартингальной меры $Q_{\lambda_{+}}$или $Q_{\lambda_{-}}$(см. замечание 1). Соответствующий выбор мартингальной меры определяется табличами 7 и 8. Каждая нижняя справедливая иена определяет соответствуючую нижнюю хеджирующую стратегию $\underline{\sigma}(k)=\left(\underline{\beta}_{k}, \underline{\gamma}_{k, 1}, \underline{\gamma}_{k, 2}\right)$, которая выбирается в соответствии с таблицами 7 и 8 . Каждая клетка таблии 7 и 8 содержит пару (верхняя/нижняя мартингальная мера; нижняя хеджирующая стратегия) для каждой возможной ситуаиии.

(б) Если платежная функция $f$ такова, что $d_{f}(s)=0$ для любого $s \in \mathbf{R}_{+}^{2}$, то соотношение (4.10) определяет единственную верхнюю справедливую цену платежного обязательства $X$ в момент $k, k=0,1, \ldots, n-1$, и результат не зависит от 
Таблица 8.

\begin{tabular}{|c|c|c|c|}
\hline & $p_{1}+p_{2}<1$ & $p_{1}+p_{2}>1$ & $p_{1}+p_{2}=1$ \\
\hline $\begin{array}{c}f \text { положительно } \\
\text { определена }\end{array}$ & $\left(Q_{\lambda_{-}} ; \sigma^{++}(k)\right)$ & $\left(Q_{\lambda_{-}} ; \sigma^{--}(k)\right)$ & $\left(Q_{\lambda_{-}} ; \alpha \sigma^{++}(k)+(1-\alpha) \sigma^{--}(k)\right)$ \\
& & $0 \leqslant \alpha \leqslant 1$ \\
\hline
\end{tabular}

выбора мартингальной меры $Q_{\lambda_{+}}$или $Q_{\lambda_{-}}$. Соответствующая единственная хеджирующая стратегия в момент $k$ равна

$$
\sigma^{++}(k)=\sigma^{+-}(k)=\sigma^{-+}(k)=\sigma^{--}(k) .
$$

Следствие 2. Из части (б) теоремы 4 и части (б) теоремы 5 очевидно следует, что если $d_{f}=0$ для любого $s \in \mathbf{R}_{+}^{2}$, то платежное обязательство $X=f^{\left(s^{(n)}\right)}$ достижимо при использовании единственной хеджирующей стратегии

$$
\sigma^{++}(k)=\sigma^{+-}(k)=\sigma^{-+}(k)=\sigma^{--}(k), \quad k=0,1, \ldots, n-1 .
$$

Единственная справедливая цена платежного обязательства $Х$ в момент $k$ определяется соотношением (4.10) (или, что эквивалентно, соотношением (4.11)), и результат не зависит от выбора мартингальной меры $Q_{\lambda_{+}}$или $Q_{\lambda_{-}}$.

\section{3. Арбитраж на динамическом рынке}

Для каждого шага двумерного бинарного рынка с горизонтом $n$ мы нашли интервал справедливых цен $(\underline{x}(k), \bar{x}(k))$. Как и в одношаговой ситуации, этот интервал открыт, так что его концы являются точками арбитража. В самом деле, нижняя и верхняя границы интервала $\underline{x}(k)$ и $\bar{x}(k)$ получаются как математические ожидания по отношению к мере $Q^{(n-k)}$ (см. (4.8)). Поскольку соответствуюшие одношаговые меры $Q_{\lambda_{+}}$и $Q \lambda_{-}$являются мартингальными мерами, мера $Q^{(n-k)}$ также является мартингальной мерой на $\tilde{\Omega}^{(n-k)}$. Однако она не является нейтральной к риску мерой, поскольку $Q_{\lambda_{+}}$(как и $Q_{\lambda_{-}}$) не является эквивалентной мерой для одношагового рынка, а значит, на рассматриваемом рынке существуют арбитражные возможности.

Рассмотрим конкретный случай арбитражной ситуации. Пусть функция $f$ положительно определена и $p_{1}<p_{2}$. Согласно теореме 4, последовательность верхних справедливых цен платежного обязательства $X$ задается соотношением (4.10), где $Q_{\lambda_{+}}$является соответствуюшей одношаговой мартингальной мерой. Если в момент 0 инвестор продает платежное обязательство $X$ по цене $\bar{x}(0)$, а затем использует стратегию $\sigma^{+-}(0)$, то в следующий момент $k=1$ стоимость портфеля будет равна

$$
x(1)=\beta_{0}^{+-} b_{0} \rho+\gamma_{0,1}^{+-} s_{0,1} \xi_{1,1}+\gamma_{0,2}^{+-} s_{0,2} \xi_{1,2}
$$

По построению, если вектор скачков $\xi=\left(\xi_{1,1} \xi_{1,2}\right)$ принимает значения $\left(u_{1}, u_{2}\right),\left(d_{1}, u_{2}\right)$ или $\left(d_{1}, d_{2}\right)$, то стоимость портфеля в момент $k=1$ равна верхней справедливой цене в момент $k=1$, то есть, $x(1)=\bar{x}(1)$. Если вектор скачков принимает значение $\left(u_{1}, d_{2}\right)$, то существует положительная разность $\bar{\delta}_{1}$ между стоимостью портфеля $x(1)$ и следующей верхней справедливой ценой $\bar{x}(1)$, равная

$$
\bar{\delta}_{1}=x(1)-\bar{x}(1)=d_{f(1)}>0 .
$$


Повторяя эти рассуждения для дальнейших моментов времени $k=2, \ldots, n$, получаем последовательность безрисковых доходов $\bar{\delta}_{k}=x(k)-\bar{x}(k)$. При этом $\bar{\delta}_{k}=d_{f(k)}>0$, если $\xi^{(k)}=\left(u_{1}, d_{2}\right)$, и $\bar{\delta}_{k}$ равно нулю в противном случае при всех $k$.

Легко проверить, что для всех других случаев, фигурирующих в теореме 4, за исключением случая, когда $d_{f}=0$ для всех $s \in \mathbf{R}_{+}^{2}$, имеет место аналогичная ситуация: величина $\bar{\delta}_{k}$ равна нулю, если вектор скачков в момент $k$ принимает три значения из четырех возможных, а в оставшемся случае инвестор получает безрисковый доход

$$
\bar{\delta}_{k}=\left|d_{f(k)}\right|,
$$

который реализует арбитражную возможность для продавца платежного обязательства.

Инвестируя одношаговые доходы $\bar{\delta}_{k}$ под процентную ставку $(\rho-1) \cdot 100 \%$, инвестор получает в момент исполнения обязательства суммарный безрисковый доход

$$
\bar{\Delta}_{n}=\bar{\delta}_{1} \rho^{n-1}+\bar{\delta}_{2} \rho^{n-2}+\ldots+\bar{\delta}_{n} .
$$

Если инвестор продает платежное обязательство $X$ в момент $k=0$ по нижней справедливой цене, а затем применяет соответствующую нижнюю хеджирующую стратегию, то получается обратная ситуация. В каждый момент времени, если вектор скачков принимает одно конкретное значение из четырех возможных, инвестор несет потери величиной

$$
\underline{\delta}_{k}=\bar{x}(k)-x(k)=\left|d_{f}(k)\right| .
$$

Суммарные накопленные потери в момент $n$ равны

$$
\underline{\Delta}_{n}=\underline{\delta}_{1} \rho^{n-1}+\underline{\delta}_{2} \rho^{n-2}+\ldots+\underline{\delta}_{n} .
$$

Это соответствует арбитражной возможности для покупателя обязательства $X$. В самом деле, предположим, что покупатель приобретает платежное обязательство $X$ в момент $k=0$ по нижней справедливой цене и финансирует покупку, продав нижний хеджирующий портфель. В каждый момент времени, если вектор скачков принимает одно соответствующее значение из четырех возможных, покупатель обязательства получает доход величиной (4.14). Суммарный накопленный доход в момент исполнения обязательства задается соотношением (4.15).

\section{5. Обобщение на случай прямоугольного носителя для распределения вектора скачков и выпуклой платежной функции}

Обобщим рассмотренную двумерную бинарную модель на случай, когда вектор скачков цен акций $\xi^{(k)}=\left(\xi_{k, 1}, \xi_{k, 2}\right), k=1, \ldots, n$, принимает значения на прямоугольнике

$$
R=\left\{y=\left(y_{1}, y_{2}\right): d_{i} \leqslant y_{i} \leqslant u_{i}, i=1,2\right\} .
$$

Будем ссылаться на эту модель, как на обобщенную двумерную бинарную модель (ОДБмодель). Очевидно, что ОДБ-рынок является неполным. Наша цель - найти верхнюю и нижнюю границы интервала справедливых цен для европейского платежного обязательства $X$ с платежной функцией $f: X=f\left(s^{(n)}\right)$. 
Замечание 5. Пусть $f-$ выпуклая функция из $\mathbf{R}^{2}$ в $\mathbf{R}^{1}$, и пусть функции $f^{(k)}$, $k=0,1, \ldots, n-1$, определены соотношением (4.4) с $f^{(n)}=f$. Тогда из (4.5) вытекает, что $f^{(k)}$ также является выпуклой функцией при $k=0,1, \ldots, n-1$, поскольку $f^{(k)}$ - выпуклая комбинация выпуклых функций.

Теорема 6. Пусть $X=f\left(s^{(n)}\right)$-европейское платежсне обязательство на ОДБ-рынке с горизонтом п. Предположим, что платежная функиия $f$ выпукла. Тогда верхняя справедливая иена обязательства $X$, а также верхние хеджирующие стратегии определяются согласно теореме 4. Нижняя справедливая цена обязательства $X$ задается формулой

$$
\underline{x}(k)=\rho^{-(n-k)} f\left(\rho^{n-k} s^{(k)}\right), \quad k=0, \ldots, n-1 .
$$

Доказательство. Начнем с одношагового ОДБ-рынка с начальным моментом времени $k=n-1$ и датой исполнения $k=n$.

Рассмотрим сначала верхнюю справедливую цену и верхнюю хеджирующую стратегию. Соответствующая задача выпуклого программирования (задача ВП) формулируется следующим образом:

$$
\text { минимизировать } \beta b_{n-1}+\gamma_{1} s_{n-1,1}+\gamma_{2} s_{n-1,2}
$$

при условии

$$
\begin{aligned}
\beta b_{n-1} \rho+\gamma_{1} s_{n-1,1} \xi_{n, 1}+\gamma_{2} s_{n-1,2} \xi_{n, 2} & \geqslant f\left(s_{n-1,1} \xi_{n, 1}, s_{n-1,2} \xi_{n, 2}\right) \\
\xi^{(n)} & =\left(\xi_{n, 1}, \xi_{n, 2}\right) \in R .
\end{aligned}
$$

Целевая функция (5.2) отвечает стоимости хеджирующего портфеля в момент $k=n-1$, a ограничения, определяющие допустимую область (5.3), отвечают ограничениям, которые гарантируют, что инвестор в момент $k=n$ сумеет исполнить обязательства с вероятностью 1.

По аналогии с доказательством теоремы 4 выберем один из случаев из таблиц 5 или 6 и докажем теорему 6 для этого случая. Остальные случаи рассматриваются аналогично.

Предположим, что платежная функция $f$ положительно определена в смысле определения 3 , и пусть $p_{1}<p_{2}$. Предположим, что теорема 4 дает решение сформулированной выше задачи ВП (5.2)-(5.3). Тогда верхняя справедливая цена платежного обязательства $X$ в момент $k=n-1$ равна

$$
\bar{x}(n-1)=f^{(n-1)}\left(s^{(n-1)}\right)
$$

где $f^{(k)}$ задается формулой (4.4) с параметрами $\left(q_{1}, q_{2}, q_{3}, q_{4}\right)=\left(p_{1}, 0, p_{2}-p_{1}, 1-p_{2}\right)$, которые являются компонентами верхней мартингальной меры $Q_{\lambda_{+}}$, выбранной для случая $p_{1}<p_{2}$. Соответствующий верхний хедж имеет вид

$$
\bar{\sigma}(n-1)=\left(\beta_{n-1}^{+-}, \gamma_{n-1,1}^{+-}, \gamma_{n-1,2}^{+-}\right)
$$

(см. формулы для $\gamma_{k, 1}^{+-}, \gamma_{k, 2}^{+-}$и $\beta_{k}^{+-} \cdot k=0,1, \ldots, n-1$, в разделе 4.1). Тогда стоимость верхнего хеджирующего портфеля в момент $k=n-1$ равна

$$
\pi(n-1)=\beta_{n-1}^{+-} b_{n-1}+\gamma_{n-1,1}^{+-} s_{n-1,1}+\gamma_{n-1,2}^{+-} s_{n-1,2} .
$$

$\mathrm{B}$ момент $k=n$ стоимость портфеля изменяется и становится равной

$$
\pi(n)=\beta_{n-1}^{+-} b_{n-1} \rho+\gamma_{n-1,1}^{+-} s_{n-1,1} \xi_{n, 1}+\gamma_{n-1,2}^{+-} s_{n-1,2} \xi_{n, 2} .
$$


Напомним, что в случае ОДБ-рынка предполагается, что вектор скачков $\xi^{(n)}$ принимает любые значения внутри прямоугольника $R$, а не только значения, соответствующие его вершинам $\left(u_{1}, u_{2}\right),\left(u_{1}, d_{2}\right),\left(d_{1}, u_{2}\right)$ и $\left(d_{1}, d_{2}\right)$.

Подставляя формулы из раздела 4.1 для компонент $\gamma_{n-1,1}^{+-}, \gamma_{n-1,2}^{+-}$и $\beta_{n-1}^{+-}$в последнюю формулу для $\pi(n)$, получаем

$$
\pi(n)=\frac{\xi_{n, 1}-d_{1}}{u_{1}-d_{1}} f_{++}^{(n-1)}+\left(\frac{\xi_{n, 2}-d_{2}}{u_{2}-d_{2}}-\frac{\xi_{n, 1}-d_{1}}{u_{1}-d_{1}}\right) f_{-+}^{(n-1)}+\frac{u_{2}-\xi_{n, 2}}{u_{2}-d_{2}} f_{--}^{(n-1)},
$$

где $f_{++}^{(k)}, f_{-+}^{(k)}$ и $f_{--}^{(k)}$ задаются соотношением (4.6). Стоимость портфеля (5.6) является линейной функщией от $\left(\xi_{n, 1}, \xi_{n, 2}\right)$, которая определяет плоскость $\pi(n)=\pi_{n}\left(\xi_{n, 1}, \xi_{n, 2}\right)$, проходящую через точки $\left(u_{1}, u_{2}, f_{++}^{(n-1)}\right),\left(d_{1}, u_{2}, f_{-+}^{(n-1)}\right)$ и $\left(d_{1}, d_{2}, f_{--}^{(n-1)}\right)$. Далее, легко проверить, что точка $\left(u_{1}, d_{2}, f_{+-}^{(n-1)}\right)$ лежит под плоскостью. В самом деле,

$$
\pi_{n}\left(u_{1}, d_{2}\right)=f_{++}^{(n-1)}-f_{-+}^{(n-1)}+f_{--}^{(n-1)}>f_{+-}^{(n-1)}
$$

в силу того факта, что $f^{(n-1)}$ положительно определена.

Определим теперь новую функцию $h$, полагая

$$
h\left(\xi_{n, 1}, \xi_{n, 2}\right)=f\left(s_{n-1,1} \xi_{n, 1}, s_{n-1,2} \xi_{n, 2}\right),
$$

где $f$ - платежная функция обязательства $X$. Функция $h$ выпукла на прямоугольнике $R$, поскольку $f$ выпукла на $\mathbf{R}^{2}$. Выпуклая поверхность $h\left(\xi_{n, 1}, \xi_{n, 2}\right)$ имеет с плоскостью $\pi_{n}\left(\xi_{n, 1}, \xi_{n, 2}\right)$ три общие точки $\left(u_{1}, u_{2}, f_{++}^{(n-1)}\right),\left(d_{1}, u_{2}, f_{-+}^{(n-1)}\right)$ и $\left(d_{1}, d_{2}, f_{-}^{(n-1)}\right)$ и проходит через точку $\left(u_{1}, d_{2}, f_{+-}^{(n-1)}\right)$, которая лежит ниже плоскости. Это говорит о том, что для всех точек $\left(\xi_{n, 1}, \xi_{n, 2}\right) \in\left(d_{1}, u_{1}\right) \times\left(d_{2}, u_{2}\right)$ поверхность $h$ лежит ниже плоскости $\pi_{n}$.

Таким образом, мы пришли к выводу, что если вектор скачков $\xi^{(n)}$ принимает одно из экстремальных значений $\left(u_{1}, u_{2}\right),\left(d_{1}, u_{2}\right)$ или $\left(d_{1}, d_{2}\right)$, то стоимость портфеля $\pi(n)$ равна терминальному значению платежной функции $f\left(s^{(n)}\right)$; если $\xi^{(n)}$ принимает любое другое значение в прямоугольнике $R$, то $\left.\pi(n)>f^{(n)}\right)$. Таким образом, мы нашли явное решение задачи ВП (5.2)-(5.3).

Можно повторить приведенные рассуждения для ОДБ-рынка с начальным моментом $k=n-2$, моментом исполнения $k=n-1$ и платежной функцией $f^{(n-1)}$.

Последовательное уменьшение начального момента времени вплоть до значения $k=0$ завершает доказательство первой части теоремы 6.

Вернемся к одношаговому ОДБ-рынку с начальным моментом $k=n-1$ и моментом исполнения $k=n$. Переключимся на нижние справедливые цены и нижние хеджирующие стратегии. Соответствуюшая задача ВП формулируется следующим образом:

$$
\text { максимизировать } \beta b_{n-1}+\gamma_{1} s_{n-1,1}+\gamma_{2} s_{n-1,2}
$$

при условии

$$
\begin{aligned}
\beta b_{n-1} \rho+\gamma_{1} s_{n-1,1} \xi_{n, 1}+\gamma_{2} s_{n-1,2} \xi_{n, 2} & \leqslant f\left(s_{n-1,1} \xi_{n, 1}, s_{n-1,2} \xi_{n, 2}\right), \\
\xi^{(n)} & =\left(\xi_{n, 1}, \xi_{n, 2}\right) \in R t .
\end{aligned}
$$

Целевая функция (5.8) отвечает стоимости хеджирующего портфеля в момент $k=n-1$, а ограничения, определяющие допустимую область (5.9), отвечают ограничениям, которые 
гарантируют, что в момент $k=n$ инвестор может выполнить контракт с положительной вероятностью.

Предположим, что вектор скачков $\xi^{(n)}$ принимает тривиальное значение $(\rho, \rho)$. Перепишем ограничение (5.9) в виде равенства

$$
\rho\left(\beta b_{n-1}+\gamma_{1} s_{n-1,1}+\gamma_{2} s_{n-1,2}\right)=f\left(\rho s^{(n-1)}\right) .
$$

Решая последнее уравнение относительно $\beta$ (считая пока значения $\gamma_{1}$ и $\gamma_{2}$ произвольными), получаем

$$
\beta=\frac{1}{b_{n-1}}\left(\frac{1}{\rho} f\left(\rho s^{(n-1)}\right)-\gamma_{1} s_{n-1,1}-\gamma_{2} s_{n-1,2}\right) .
$$

Тогда для любого $\xi^{(n)} \in R$ стоимость хеджируюшего портфеля в момент $k=n$ равна

$$
\pi(n)=f\left(s_{n-1,1} \rho, s_{n-1,2} \rho\right)+\gamma_{1} s_{n-1,1}\left(\xi_{1}-\rho\right)+\gamma_{2} s_{n-1,2}\left(\xi_{2}-\rho\right) \text {. }
$$

Мы хотим показать, что для любого $\xi^{(n)} \in R$ справедливо неравенство

$$
\pi(n) \leqslant f\left(s_{n-1,1} \xi_{n, 1}, s_{n-1,2} \xi_{n, 2}\right) .
$$

Используем выпуклую на $R$ функцию $h$, определенную в соотношении (5.7). Неравенство (5.12) можно записать в виде

$$
h(\rho, \rho)+\gamma_{1} s_{n-1,1}\left(\xi_{1}-\rho\right)+\gamma_{2} s_{n-1,2}\left(\xi_{2}-\rho\right) \leqslant h\left(\xi_{n, 1}, \xi_{n, 2}\right) .
$$

Поскольку $h$ выпукла на $R$, для каждой точки из $R$ сушествует опорная гиперплоскость. Пусть

$$
l\left(y_{1}, y_{2}\right)=c_{1}\left(y_{1}-\rho\right)+c_{2}\left(y_{2}-\rho\right)+h(\rho, \rho)
$$

- опорная гиперплоскость, отвечающая точке $(\rho, \rho) \in R$. Легко проверить, что

$$
h(\rho, \rho)=l(\rho, \rho)
$$

Тогда, по определению, для любого $\left(y_{1}, y_{2}\right) \in R$ справедливо неравенство

$$
h\left(y_{1}, y_{2}\right) \geqslant l\left(y_{1}, y_{2}\right) \text {. }
$$

Положим

$$
\gamma_{1}=\frac{c_{1}}{s_{n-1,1}}, \quad \gamma_{2}=\frac{c_{2}}{s_{n-1,2}}
$$

Тогда соотношения (5.14), (5.10) определяют оптимальную нижнюю хеджирующую стратегию, а нижняя справедливая цена обязательства $X$ в момент $k=n-1$ задается оптимальным значением целевого функционала (5.8)

$$
\underline{x}(n-1)=\rho^{-1} f\left(\rho s^{(n-1)}\right) .
$$

Обратная индукция по времени завершает доказательство второй части теоремы 6. 


\section{6. Заключительные замечания}

1. Как мы видели, простейший бинарный рынок является неполным, если число акций больше единицы. Тем не менее, это не так в случае непрерывного времени. Рынок в многомерной модели Блэка-Шоулса является полным (см., например, [2]). Это говорит о том, что постановка задачи в дискретном времени дает возможность изучать некоторые интересные эффекты, которые нельзя наблюдать в непрерывном времени.

2. Стратегия торговли называется самофинансируемой, если изменение содержания портфеля не влияет на его стоимость, то есть не разрешено потребление или экзогенный доход. Верхние и нижние хеджирующие стратегии, полученные в этой работе, в общем, не являются самофинансируемыми. Как было показано в разделе 4.3, если опцион продается по верхней справедливой цене и продавец применяет верхнюю хеджирующую стратегию, то он в каждый момент получает ненулевой безрисковый доход с ненулевой вероятностью. Предполагается, что эти дополнительные средства изымаются из портфеля, что делает такую стратегию полу-самофинансируемой. Если опцион продается по нижней справедливой цене и продавец применяет соответствующую нижнюю хеджирующую стратегию, то он в каждый момент с ненулевой вероятностью несет ненулевые потери. Соответствующие средства должны добавляться в портфель инвестора каждый раз, когда происходят потери, так что нижняя хеджирующая стратегия также является полу-самофинансируемой. Каждый раз, когда продавец опциона несет потери, покупатель опциона получает равное количество денег. Если опцион продается по любой другой цене внутри интервала справедливых цен, то продавец и покупатель в каждый момент делят между собой доходы и потери. Эта идея получила теоретическое развитие в $[5,6]$. Мы планируем развить теоретический и вычислительный подходы для случая рассмотренного здесь двумерного рынка.

3. Мы планируем дальнейшее изучение случая выпуклого носителя для распределения вектора скачков цен акций и выпуклой платежной функции. Случай марковской зависимости между векторами скачков цен, а также случай неодинаково распределенных векторов скачков являются предметом дальнейших исследований.

\section{Благодарности}

Мы благодарны Норму Джозефи за интересные и полезные обсуждения и замечания, а также за внимательное чтение рукописи. Второй автор выражает признательность за финансовую поддержку исследовательскому фонду ERM Бентли колледжа, а также Фонду Александра Гумбольдта за финансовую поддержку. Второй автор благодарит также Серджио Альбеверио и университет Бонна за гостеприимство.

Статья была почти закончена, когда Александр Нагаев трагически погиб. Ответственность за окончательную редакцию статьи и за возможные недостатки полностью лежит на втором авторе. 


\section{Список литературы}

1. Cox J. C., Ross R. A., Rubinstein M., Option pricing: a simplified approach. J. Finan. Economics (1976) 7, 229-263.

2. Karatzas I., Lectures on the mathematics of finance. Amer. Math. Soc., Providence, 1996.

3. Motoczynski M., Stettner L., On option pricing in the multidimensional Cox-Ross-Rubinstein model. Appl. Mathematicae (1998) 25, 55-72.

4. Nagaev A. V., Nagaev S. A., A diffusion approximation for the riskless profit under selling of discrete time call options. Appl. Mathematicae (2003) 30. 173-191.

5. Nagaev A. V., Nagaev S. A., Kunst R. M., A diffusion approximation for the riskless profit under selling of discrete time call options. Non-identically distributed jumps. Preprint, Institute for Advanced Studies, Vienna, 2005.

6. Nagaev A. V., Nagaev S. A., Kunst R. M., A diffusion approximation to the Markov chains model of the financial market and the expected riskless profit under selling of call and put options. Preprint, Institute for Advanced Studies, Vienna, 2005.

7. Pliska S. R., Introduction to mathematical finance. Discrete time models. Blackwell, Oxford, 1997.

8. Tessitore G., Zabczyk J., Pricing options for multinomial models. Bull. Polish Acad. Sci. Math. (1996) 44, 363-380.

Статья поступила 15.06.2005. 